\title{
Epigenetic mechanisms in pathogenesis of multiple sclerosis
}

\begin{abstract}
The etiology of multiple sclerosis remains largely unknown. It has a complex pathogenesis and likely a multifactorial etiology. The current paradigm for understanding its development is that MS is triggered by the exposure to certain environmental factors, particularly, viruses in genetically-susceptible individuals. Although both genetic and environmental components significantly contribute to disease susceptibility, their effects on the immune response could be mediated by changes in epigenetic regulation. Molecular mechanisms through which the impact of the environmental factors is translated into the changes in gene expression are represented by DNA methylation, post-translational histone modification and noncoding RNAs. These mechanisms are regulated by tissue selective and cell specific enzymes, while at the same time they regulate the gene expression without affecting the DNA sequence. It opens new possibilities in studying the relationship between the genes and environmental factors in multiple sclerosis. This article will review the current understanding of the epigenetic mechanisms in multiple sclerosis.
\end{abstract}

Keywords: multiple sclrerosis, epigenetics, DNA methylation, histone modification, non-coding RNAs
Volume 2 Issue 2 - 2018

\author{
Goldina IA, Gaidul KV, Kozlov VA \\ Scientific Research Institute of Fundamental and Clinical \\ Immunology, Russia
}

Correspondence: Goldina Irina Alexandrovna, Scientific Research Institute of Fundamental and Clinical Immunology, Novosibirsk, Russia, Email igoldina@mail.ru

Received: March 01, 2018 | Published: March 29, 2018
Abbreviations: MS, multiple sclerosis; TET, ten-eleven translocation; SNP, single nucleotide polymorphisms; PAD, polymorphisms peptidylarginine deiminase; TGFBR, transforming growth factor beta receptor; EAE, experimental autoimmune encephalomyelitis

\section{Introduction}

Multiple sclerosis (MS) is a chronic autoimmune disease of the central nervous system with immune induced myelin destruction followed by multifocal demyelination of the nervous tissues. ${ }^{1}$ MS is the most widely spread among the human diseases characterized by myelin destruction.$^{2}$ Until now both the etiology and pathogenesis of MS have to a large extent remained unclear. Substantial efforts have been made over the last decades to identify biomarkers for MS that can identify disease diagnosis, predict disease progression, and improve clinical outcomes. Degenerative and inflammatory changes in the nervous tissue in MS are characterized by a multiple fibrosis of neuron myelin sheath accompanied by the formation of sclerotic plaques and axon pathology resulting in progressive neurological dysfunction. ${ }^{3,4}$ Based on the pathomorphological aspects of the disease, French neurologist Dr. Jean-Martin Charcot was the first to recognize MS as a distinct disease in the end of the 19th century. He described the clusters of inflammatory cells in the perivascular space in the brain and spinal cord in patients with intermittent neurological disorders. ${ }^{5}$

Considering the current body of knowledge about MS etiology, it is believed that the disease appears in genetically predisposed individuals under the influence of some trigger factors in the environment. Their nature and the multiplicity of their impact during the prenatal development, childhood and adolescence are decisive for the disease development. ${ }^{6,7}$ Complex interaction between genetic predisposition and environmental factors determines the development of MS as a polyetiologic immune-mediated disease. ${ }^{8}$ In its pathogenesis, malfunction of regulatory T-cells plays the key role, while under normal conditions these cells suppress the activation of TH-I and TH-17 effector cells. ${ }^{9}$ It is believed that the disease manifestation is connected with the migration of autoreactive lymphocytes through the hematoencephalic barrier. Autoreactive T-cell-mediated immune response to myelin antigens causes the inflammation followed by axonal demyelination of neurons and neurological disorders. ${ }^{10,11}$ The interplay of environmental influences and individual genetic susceptibility modulates disease presentation and therapeutic responsiveness.

\section{Environmental risk factors}

Such extra genetic factors as the impact of chemical substances, some drugs, excessive alcohol uptake, emotional stress, ion radiation and tobacco smoking, occupation are considered to be the ones associated with the course of MS. ${ }^{12-17}$ At the same time, it is supposed that a lack of vitamin $\mathrm{D},{ }^{18-21}$ the influence of viruses and the protozoa, such as herpes type IV, ${ }^{22}$ Chlamydia pneumonia ${ }^{23}$ the Epstein-Barr virus $^{24}$ as well as mobile elements of the genome represented by human endogenous retroviruses ${ }^{25-28}$ can trigger the pathological process and are considered the most significant factors in the etiology of MS. Moreover, there is a growing body of evidence suggesting that the intestine microbiota can play both pathogenic and protective roles in MS disease progression. Thus, in experimental autoimmune encephalomyelitis (EAE), the animal model of MS, it has been shown that intestinal microorganisms regulate the polarization of $T$ helper cells from Th1-Th17 up to Th2, the function of regulatory T cells, and the activity of B cells; they participate in the pathogenesis of EAE and contribute to its prevention and treatment. ${ }^{29}$

\section{Epigenetic changes in MS}

Epigenome is an inherited combination of mechanisms determining gene expression..$^{30}$ It is changeable during the life of an individual, sensitive to the influence of environmental factors and changeable in the course of the disease while at the same time being different 
in individuals with identical genomes. ${ }^{31}$ The term "epigenetics" has evolved to define mechanisms underlying phenotype plasticity due to environmental influences, parent-of-origin effects, gene-dosage control, imprinting, and X-chromosome inactivation. The molecular mechanisms through which environmental signals are translated into changes in gene expression are represented by DNA methylation, post-translational modification of nucleosomal histones, and noncoding RNAs. These mechanisms are regulated by families of specialized enzymes that are tissue - selective and cell-type specific. As regards immunocompetent cells, these mechanisms are regulated by tissue-selective enzymes, in particular, central nervous system selective and cell specific enzymes. ${ }^{32,33}$ They regulate gene expression without affecting the DNA sequence, thus opening new perspectives in investigating the relationship between the genes and environmental factors in MS. ${ }^{34,35}$

Epigenetic mechanisms play a very important role in MS etiology and pathogenesis as they modulate a range of biological processes, such as X-chromosome inactivation, myelin production and formation of the T-specific immune response. ${ }^{36-38}$ Based on the current understanding of gene expression, eukaryotes differ from prokaryotes by a considerably larger number of genes, thus making it necessary to pack genes into an ultrastructure known as chromatin inside the cell nucleus via histones. It is known that in its native form chromatin is very compact and cannot be influenced by transcription factors due to its inaccessibility. Eukaryotic cells use such chromatin structure not only to pack DNA but also to prevent the expression of the genes which are not necessary for cell functions but for which the cell expresses the transcription factors connected with recognizing the elements inside the genome known as "transcriptional noise". ${ }^{39,40}$ The main chromatin modifications are represented by DNA methylation and post translational modification of histones.

\section{Histone modifications}

Histone octamer system consisting of two copies of each of the four proteins $\mathrm{H} 2 \mathrm{~A}, \mathrm{H} 2 \mathrm{~B}, \mathrm{H} 3, \mathrm{H} 4$ is capable of changing the spatial structure of chromatin and its accessibility to transcription factors. Transcription is regulated through DNA methylation of cytosine residues which inhibits the transcription of the genetic chain. ${ }^{41}$ Histone protein modification, such as acetylation, methylation, phosphorylation, ubiquitination, sumoylation (citrullination), takes place at the post-translational level and represents so-called histone code which initiates the process of chromatin remodeling in a less compact structure. It can be affected by the transcription factors and results in activation or repression of genes associated with the modified histone. ${ }^{42}$ Histone modification process can be affected by the environmental factors and is considered as a nonhereditary mechanism in gene regulation instability. ${ }^{43}$ At the same time, based on single nucleotide polymorphisms (SNP) - rs 2522129, rs 2675231, rs 2389963 , such authors as ${ }^{44}$ have detected gene variants from HDAC family whose presence testifies to a high risk of MS development. ${ }^{45}$ It was discovered that MBP citrullination by the enzyme PAD-2 plays an important role in MS pathophysiology. For example, the level of citrullinated MBP in patients with MS considerably exceeds that in healthy humans and the Alzheimer's disease patients. Citrullinated MBP is less stable than the non-modified one, thus this process can result in myelin destruction by an autoimmune response to MBP. ${ }^{46}$ In MS, hypermethylation of histone $\mathrm{H} 3$ protein has been detected in oligodendrocytes of chronic lesions, while at the early stages of the pathological process in MS H3 deacetylation has been recorded. Thus, $\mathrm{H} 3$ acetylation level correlates with an increased duration of the disease and the decreased remyelinization intensity in MS patients. ${ }^{47}$ the decreased histone acetylation and increased DNA methylation in oligodendrocyte lineage cells enhance myelin repair, which is beneficial for MS, while the same epigenetic processes in T cells augment their pro-inflammatory phenotype, which can exacerbate disease severity. ${ }^{48}$

\section{Changes in DNA methylation}

DNA methylation is an epigenetic mechanism carried out by the enzyme DNA methyltransferase (DNMT), which transfers methyl groups to cytosine residues in regulatory gene regions. The processes of DNA methylation and histone modifications are interrelated. ${ }^{49}$ Hypomethylation which presents a disorder in DNA - methylation and histone modifications under the influence of environmental factors results in a considerable level of disruption in gene regulation, including active transcription, and breakdown in tolerance to own antigens in MS, while DNA hypermethylation and histone hypoacetylation condition induce gene inactivation. ${ }^{50,51}$ Demethylation is achieved by the ten-eleven translocation (TET) enzymes, which catalyze hydroxy-methylation, formylation, and carboxylation of cytosine residues, or by the DNA excision-repair system after 5-methylcytosine or 5-hydroxymethylcytosine is converted into thymine or 5-hydroxymethyluracil, catalyzed by the AID/APOBEC family of enzymes. ${ }^{52,53}$ DNA methylation profile differs in distinct individuals, is inherited and determines the gene expression profile. ${ }^{54,55}$ DNA methylation in mammals is predominantly carried out in 5'-cytosin - guanine-3' dinucleotides, CpG-islands consisting of 500 nucleotide pairs with CG content greater than 55\%, predominately located in promoter gene regions. ${ }^{56}$ It is the most thoroughly studied epigenetic mechanism of normal development, genome stability and cell proliferation.

No proof has been obtained that DNA methylation at HLA-DRB1 and HLA-DRB5 5 allele's influences the phenotypic heterogeneity in MS. ${ }^{6}$ At the same time, a considerable decrease in the level of cytosine methylation in MS has been observed in $\mathrm{CpG}$ islands in neurons. In addition, an increased activity of demethylase ${ }^{57}$ and hypomethylation have been detected in the promoter region of gene enzyme peptidylarginine deiminase (PAD-2) which results in an increase in gene expression of this enzyme and arginine conversion to citrullin in the myelin basic protein (the reaction of deimination). In its turn, it leads to irreversible changes in its biological properties, in particular, its greater sensitivity to the impact of proteolytic ferments and impairment in its ability to interact with the lipid layer, which determines myelin instability in MS as well as the formation of deiminated membrane-bound MBP as immune dominant epitope. ${ }^{58}$ It follows that MBP citrullination is regulated by DNA methylation. Such changes in the MBP structure and its properties at the early stages of pathogenic process in MS result in sensibilisation and the formation of autoreactive T-cells clones as well as chronic inflammatory response. ${ }^{59}$ In addition, it has been established that the quantity of PAD-2 in the brain of MS patients and that of citrullinated myelin is considerably increased, while myelin instability presents one of the demethylation mechanisms in MS PC..$^{46}$ Along with myelin destruction, hypomethylation in the promoter region of IL-17 gene has been also detected in MS patients accompanied by a considerable increase in T-cells differentiation to the TH17 phenotype. In order to 
identify DNA methylation changes in $\mathrm{CD}^{+} \mathrm{T}$ cells in patients with relapsing-remitting (RR-MS) and secondary-progressive (SP-MS) disease and healthy controls (HC), DNA methylation analysis in $\mathrm{CD}^{+} \mathrm{T}$ cells from RR-MS, SP-MS, and HC and associated identified changes with the nearby risk allele, smoking, age, and gene expression have been performed by the. ${ }^{60}$

They observed significant methylation differences in the VMP1/ MIR21 locus, with RR-MS displaying higher methylation compared to SP-MS and HC. VMP1/MIR21 methylation did not correlate with a known MS risk variant in VMP1 or smoking but displayed a significant negative correlation with age and the levels of mature miR-21 in CD4 $4^{+} \mathrm{T}$ cells. Accordingly, RR-MS displayed lower levels of miR-21 compared to SP-MS, which might reflect differences in age between the groups, and healthy individuals and a significant enrichment of up-regulated miR-21 target genes. Moreover, evidence showed the hypermethylation of human leukocyte antigen-DRB1 (HLA-DRB1) in CD4 ${ }^{+} \mathrm{T}$ cells, the genome-wide DNA methylation in $\mathrm{CD}^{+} \mathrm{T}$ cells, the hypermethylation of interleukin-4 (IL-4)/forkhead winged helix transcription factor 3 (Foxp3), and the demethylation of interferon- $\gamma(\mathrm{IFN}-\gamma) / \mathrm{IL}-17 \mathrm{a}$ in CD44+ encephalitogenic T cells. Studies also showed the hypermethylation of SH2-containing protein tyrosine phosphatase-1 (SHP-1) in peripheral blood mononuclear cells (PBMCs) and methylated changes of genes regulating oligodendrocyte and neuronal function in normal-appearing white matter. ${ }^{61}$ It has been established that the mechanisms regulating DNA methylation are at the same time involved in the mechanisms of neurodegeneration mediated by the up regulation of DNMT. In particular, it has been discovered that DNMT3a activation predominant in neuron synapses and mitochondria results in DNA hypermethylation and motor neuron apoptosis, while the suppression of its catalytic activity prevents DNA methylation and apoptosis. ${ }^{62}$ It can be assumed that this cell mechanism can be used in the treatment of neurodegenerative diseases. So, DNA methylation, a significant epigenetic modification, is extensively involved in MS pathogenesis through the regulation of gene expression.

\section{MiRNA expression in MS}

MiRNAs present a class of small non-coding RNA molecules whose high level of expression is revealed in the immune and central nervous system cells which performs a very important role in cell proliferation, differentiation and apoptosis and regulates the function of up to $30 \%$ of protein-coding gene modulation at the posttranslational level. ${ }^{63,64}$ These endogenous, evolutionary stable, single chain RNAs consisting of 22-25 nucleotides control the regulation of protein-coding gene expression and cause its degradation through direct translational repression and cleavage and complementary mRNA binding. ${ }^{65,66}$ MiRNAs directly modulate the expression of regulatory proteins necessary for the normal development and functioning of the immune system, namely, maturation and functioning of granulocytes, monocytes, macrophages, dendrite cells and natural killers as well as activation and differentiation of T- and B-lymphocytes. ${ }^{67,68}$

MiRNAs specific to immunocompetent cells in humans are described. They include miR-143 in neutrophils, miR-125 in T-cells, miR-500 in monocytes, miR-150 in lymphoid cells precursors, miR 652 and miR 2239 in myeloid cells precursors whose expression has a negative correlation with the expression of their target genes while their activation inhibits the expression of genes involved in transcriptional regulation. ${ }^{69}$ Malfunction of regulation of miRNA expression is associated with oncogenesis, autoimmunity and neurodegeneration as well as myelin regeneration, gliogenesis and neurogenesis. ${ }^{70-72}$ MiRNAs regulate the self-support in neural stem cells detected in plaques in $\mathrm{MS}^{73}$ and in neurogenesis in adults ${ }^{74,75}$ which results in neuron regeneration in MS. A great number of miRNAs have been described in disorders associated with the regulation of gene expression both in blood cells, brain lesions in astrocytes and immune competent cells infiltrating sclerotic plaque formation and extracellularly occur in MS. ${ }^{76-78}$ Hence, an aberrant profile of miRNA expression in mononuclear blood cells (miR-18b, miR-599) and brain cells (miR-155, miR-34a) has been identified in MS patients. What is interesting to note is that in this case miR-155 expression increases in microphages, $\mathrm{T}$ - and $\mathrm{B}$-lymphocytes under the influence of pro-inflammatory cytokines, which attests to the involvement of this miRNA in the inflammatory process. ${ }^{79}$ According to,${ }^{80}$ miR-326 expression in the blood of MS patients increases during the acute phase of the disease and is associated with CD4+ T helpers differentiation in IL-17 generating TH-17 cells involved in the pathogenesis. MiR-326 expressed in TH-17 inhibits Ets-1, a negative regulator of TH-17 differentiation. Based on the data collected by, ${ }^{66}$ it has been established that the level of miRNA miR-145 in MS patients considerably exceeds that at healthy individuals. Authors ${ }^{76}$ have been established that miRNAs miR-155, miR-34a, miR-326 suppresses the expression of CD47 in active sclerotic plaques leading to the activation of microphages followed by myelin phagocytosis. It should be pointed out that in MS patients MiR-323 is activated in whole blood, active brain lesions and T-regulatory cells. ${ }^{64}$

When studying miRNA expression profile in blood mononuclear cells of healthy individuals and MS patients during the acute and remission phases of disease, it was established that miR-18b and miR-599 dysregulation is characteristic of the acute period, while dysregulation of miR-96 is observed during the remission phase. It's important to point out that the genes which are under the influence of miR-96 participate in the activation of cytokine production. ${ }^{81}$ Different expression profiles and cell specificity in miRNA in $\mathrm{CD}^{+}$, $\mathrm{CD} 8^{+}$T-lymphocytes and B-lymphocytes of MS patients have been identified which correlates with the expression of genes targets. ${ }^{82}$ At the same time in all MS types, miR-17 and miR-20a expression was considerably suppressed compared to that in healthy individuals. These miRNAs inhibit T-cells activation genes which are activated in MS patients ${ }^{83}$ It was also discovered that mir-155, miR-338 and miR-491 expression is increased in MS patients. They suppress aldoketoreductase (AKR1C1 and AKR1C2) translation, key enzymes involved in such neurosteroid production as allopregnanolone in the brain which decreases in the intensity of inflammation as well as axon and myelin damage. ${ }^{84}$ When analyzing the miRNA profile of the purified naïve $\mathrm{CD} 4^{+}$T-cells in order to indentify the mechanism through which they are induced to differentiation into proinflammatory phenotype in $\mathrm{MS}$, it was established that in $\mathrm{CD} 4^{+} \mathrm{T}$ naïve cells miR128 and miR-27b hyper expression is observed, while in MS patients that of miR-340 in memory T-cells. ${ }^{85}$

Along with miRNAs described in mononuclear cells, $\mathrm{CD}^{+}$, CD8 ${ }^{+}$T-cells and B-lymphocytes, miRNAs circulating in serum were detected in MS patients. They are distinguished by high stability in biological liquids, which supports the hypothesis according to which miRNAs are freed from the cell in exosomes formed by the cell membrane protecting them from blood RNAs. It was validated 
the 7 serum miRNAs that differentiate patients with MS from healthy controls: miR-320 a upregulation was the most significantly changing serum miRNA in patients with MS. It was also identified 2 miRNAs linked to disease progression, with miR-27a-3p being the most significant. Ten miRNAs correlated with the Expanded Disability Status Scale of which miR.199a.5p had the strongest correlation with disability. ${ }^{86}$ According to another data, in the serum of MS patients the extracellular miRNAs miR-614, miR-572, miR-1826, miR-422a and miR-22 are considerably activated, while the activity of miR1979 is suppressed ${ }^{63}$ Considerable increase in the levels of miR23a and miR223 in serum of MS patients compared to those in healthy controls has been established. ${ }^{87}$

One of the main issues in pathogenesis of MS is Th17/regulatory $\mathrm{T}$ cells (Treg) imbalance. There are growing interests in nominating miRNAs involved in Th17 cell differentiation, suggesting them as new therapeutic agents that may reduce in patients with MS, suppresses induction of Treg cells by targeting insulin - like growth factor 1 receptor $(I G F 1 R)$ and transforming growth factor beta receptor 1 (TGFBR1). Consistently, the expression of IGF1R and TGFBR1 on circulating naive $\mathrm{CD} 4^{+} \mathrm{T}$ cells is reduced in patients with MS. Thus, exosomal let-7i regulates MS pathogenesis by blocking the IGF1R/ TGFBR1 pathway. ${ }^{88}$ According to results, ${ }^{89}$ miR-141 and miR-200a may be key miRNAs in progression of symptoms of MS through inducing differentiation of Th17 cells and inhibiting differentiation to Treg cells. The data suggest that these miRNAs may probably inhibit negative regulators of Th17 cell differentiation, thus promoting its differentiation. Under the investigation of transcript levels of miR$27 \mathrm{a}$ and miR-214, in purified $\mathrm{CD} 4^{+} \mathrm{T}$ cells of MS patients, during relapsing and remitting phases in inducing differentiation of $T$ naïve cells to Th17 cells, the upregulation of miR27a in relapsing phase of multiple sclerosis compared to remitting phase and healthy volunteers while miR-214 downregulated in relapsing phase of MS compared to remitting phase and healthy volunteers was indicated. In silico studies demonstrated the pathways by which miR-27a and miR-214 could effect on $\mathrm{CD}^{+} \mathrm{T}$ cell lineage including TGF- $\beta$ and mTOR signaling, respectively. The data suggest that miR-27a may probably inhibit negative regulators of Th17 cell differentiation, thus promoting its differentiation while miR-214 has an adverse effect. ${ }^{90}$

These data confirm the role miRNA dependent regulatory mechanisms play in MS immunopathogenesis. Being part of epigenetic mechanism in pathogenesis of MS, circulating and cell specific miRNAs are considered as diagnostic markers as well as the targets for therapeutic intervention in MS.

\section{Conclusion}

The study of epigenetic mechanisms regulation of regulation of gene expression in MS patients has made it possible to single out changes typical for this disease and may provide an alternative approach to its better understand and manage. Histone modification, changes in the DNA methylation, hypomethylation in the promoter gene region of peptidylarginine deiminase citrullinating enzyme gene (PAD 2) result in the conversion of arginine on citrullin in MBP and damage its ability to interact with the lipid layer. It determines myelin instability in MS as well as violations in regulation of miRNA expression, and small non-coding RNA molecules controlling cell proliferation, differentiation and apoptosis regulating gene expression at the post- translational level. It is associated with myelin neurodegeneration and regeneration, gliogenesis and neurogenesis as a result of which T-cells differentiation in TH-17 phenotype intensifies. It also leads to the activation of macrophages as well as increased myelin phagocytosis and suppression of neurosteroid production which decrease the intensity of inflammation and damage to myelin and axons as well as oligodendrocytes dismaturity. The given epigenetically predetermined morphological and immunological changes result in progressive neurological dysfunction and formation of clinical presentation of MS. Thus epigenetic mechanisms of regulation of gene expression present an important component in etiopathogenesis of MS being a link between the genetic elements and environmental factors, participating in the formation of predisposition to and being responsible for the most important immunological, morphological and pathophysiological mechanisms in the pathological process. Epigenetic modifications are considered to be the mediators of gene-environment interactions and a growing body of evidence suggests that they play an important role in MS pathology and could be potential therapeutic targets. Further studies of the epigenetic mechanisms in MS will probably allow developing new criteria for differential diagnostics and monitoring disease activity as well as formulating epigenetically solid approaches to its therapy.

\section{Acknowledgment}

Author declares no acknowledgment.

\section{Conflict of interest}

The authors have no any financial interests relating to this paper.

\section{References}

1. Trapp BD, Nave KA. Multiple sclerosis: an immune or neurodegenerative disorder? Annu Rev Neurosci. 2008;31:247-269.

2. Hirtz D, Thurman DJ, Gwinn Hary K, et al. How common are the "common" neurologic disorders? Neurology. 2007;68(5):326-337.

3. Prat A, Antel J. Pathogenesis of multiple sclerosis. Curr Opin Neurol. 2005;18(3):225-230.

4. Loterotti A, Berger T, Reind M. Biological markers for multiple sclerosis. Curr Med Chem. 2007;14(18):1956-1965.

5. Charcot JM. Lecons sur les maladies du systeme nerveux faites a la Salpetriere. Paris: A Delahaye; 1880.

6. Handel AE, De Luca GC, Morahan J, et al. (2010) No evidence for an effect of DNA methylation on multiple sclerosis severity at HLADRB $1 * 15$ or HLA-DRB5. J Neuroimmunol. 223(1-2):120-123.

7. Ramagopalan SV, Dobson R, Meier UC, et al. Multiple sclerosis: risk factors, prodromes, and potential causal pathways. Lancet Neurol. 2010;9(7):727-739.

8. Ascherio A. Environmental factors in multiple sclerosis. Expert Rev Neurother. 2013;13(12 Suppl):3-9.

9. Gonsette RE. Self-tolerance in multiple sclerosis. Acta Neurol Belg. 2012;112(2):133-140.

10. Ireland S, Monson N. Potential impact of B cells and T cell function in multiple sclerosis. Mult Scler Int. 2011;423971.

11. Haegert DG. Multiple sclerosis: a disorder of altered T-cell homeostasis. Mult Scler Int. 2011;461304. 
12. Briggs FB, Acuna B, Shen L, et al. Smoking and risk of multiple sclerosis: evidence of modification by NAT1 variants. Epidemiology. 2014;25(4):605-614.

13. Horwitz H, Ahlgren B, Nærum E. Effect of occupation on risk of developing MS: an insurance cohort study. BMJ Open. 2013;3(6).

14. Benito-León J, Labiano-Fontcuberta A, Mitchell AJ, et al. Multiple sclerosis is associated with high trait anger: A case-control study. $J$ Neurol Sci. 2014;340(1-2):69-74.

15. Ceccatelli S, Tamm C, Zhang Q, et al. Mechanisms and modulation of neuronal cell damage induced by oxidative stress. Physiol Behav. 2007;92(1-2):87-92.

16. Hedström AK, Hillert J, Olsson T, et al. Alcohol as a modifiable lifestyle factor affecting multiple sclerosis risk. JAMA Neurol. 2014;71(3):300 305

17. Javierre MB. Environmental triggers and epigenetic deregulation in autoimmune disease. Discov Med. 2011;12(67):535-545.

18. Goodin DS. The causal cascade to multiple sclerosis: a model for MS pathogenesis. PLoS One. 2009;4(2):e4565.

19. Faridar A, Eskandari G, Sahraian MA, et al. Vitamin D and multiple sclerosis: a critical review and recommendation on treatment. Acta Neurologica Belgia. 2012;112(4):327-333.

20. Hatamian H, Bidabadi E, Seyed Saadat SM, et al. Is serum vitamin D levels associated with disability in patients with newly diagnosed multiple sclerosis? Iran J Neurol. 2013;12(2):41-46.

21. Christensen T. Association of human endogenous retroviruses with multiple sclerosis and possible interactions with herpes viruses. Rev Med Virol. 2005;15(3):179-211.

22. Franciotta D, Zardini E, Bergamaschi R, et al. Analysis of Chlamydia pneumoniae-specific oligoclonal bands in multiple sclerosis and other neurologic diseases. Acta Neurol Scand. 2005;112(4):238-241.

23. Ascherio A, Munger KL, White R, et al. Vitamin D as an early predictor of multiple sclerosis activity and progression. JAMA Neurol. 2014;71(3):306-314.

24. Hutchinson M. Epsteni-Barr virus is a necessary causative agent in the pathogenesis of multiple sclerosis: commentary. Mult Scler. 2013;19(13):1694-1695.

25. Garcia-Montojo M, Dominguez-Mozo M, Arias-Leal A, et al. The DNA copy number of human endogenous retrovirus-W (MSRV-type) is increased in multiple sclerosis patients and is influenced by gender and disease severity. PLoS One. 2013;8(1):e53623.

26. Curtin F, Lang AB, Perron $\mathrm{H}$, et al. GNbAC1, a humanized monoclonal antibody against the envelope protein of multiple sclerosis-associated endogenous retrovirus: a first-in-humans randomized clinical study. Clin Ther. 2012;34(12):2268-2278.

27. Goldina IA, Gaidul KV, Kozlov VA. Endogenniy retrovirus cheloveka $H E R V-E \succsim 4-1$ vpatogeneserasseiannogosclerosa: immunomoduliruushie svoistva retrovirusnogo peptida. Neuroimmunology IX. 2011;55-56.

28. Goldina IA, Gaidul KV, Kozlov VA. Indukcia expressii gena envelope endogennogo retrovirusa $H E R V-E \quad \lambda \quad 4-1 \quad \mathrm{v}$ immunocompetentnych kletkach s vysokim urovnem funkcionalnoi aktivnosti. Rossiiskii immunologicheskii Journal. 2012;6(14):63-65.

29. Castillo-Álvarez F, Marzo-Sola ME. Role of intestinal microbiota in the development of multiple sclerosis. Neurologia. 2017;32(3):175-184.

30. Petronis A. Epigenetics and twins: three variations on the theme. Trends Genet. 2006;22(7):347-350.
31. Fraga MF, Ballestar E, Paz MF, et al. Epigenetic differences arise during the lifetime of monozygotic twins. Proc Natl Acad Sci USA. 2005;102(30):10604-10609.

32. Bollatti V, Baccarelli A. Environmental epigenetics. Heredity. 2010;105(1):105-112.

33. Huynh JL, Casaccia P. Epigenetic mechanisms in multiple sclerosis: implications for pathogenesis and treatment. Lancet Neurol. 2013;12(2):195-206

34. Ptashe M. On the use of the word "epigenetic". Curr Biol. 2007;17(7):R233-236

35. Grolleau Julius A, Ray D, Yung RL. The role of epigenetics in aging and autoimmunity. Clin Rev Allergy Immun. 2010;39(1):42-50.

36. Kurtuncu M, Tuzun E. Multiple sclerosis: could it be an epigenetic disease? Med hypotheses. 2008;71(6):945-947.

37. Hewagama A, Richardson B. The genetics and epigenetics of autoimmune diseases. J Autoimmun. 2009;33(1):3-11.

38. Garden GA. Epigenetic and the modulation of neuroinflammation. Neurotherapeutics. 2013;10(4):782-788

39. Bird AP. Gene number, noise reduction and biological complexity. Trends Genet. 1995;11:94-100.

40. Li E. Chromatin modification and epigenetic reprogramming in mammalian development. Nat Rev Genet. 2002;11(3):662-673.

41. Dieker J, Muller S. Epigenetic histone code and autoimmunity. Clin Rev Allergy Immunol. 2010;11(39):78-84.

42. Berger SL. The complex language of chromatin regulation during transcription. Nature. 2007;447(7143):407-412.

43. Zhang Y, Reinberg D. Transcription regulation by histone methylation: interplay between different covalent modifications of the core histone tails. Genes Dev. 2001;15(18):2343-2360.

44. Inkster B, Strijbis EM, Vounou M, et al. Histone deacetylase gene variants predict brain volume changers in multiple sclerosis. Neurobiol Aging. 2012;34(1):238-247.

45. Zhang F, Shi Y, Wang L, et al. Role of HDAC3 on p53 expression and apoptosis in T cells of patients with multiple sclerosis. PLoS One. 2011;6(2).

46. Moscarello MA, Mastronardi FG, Wood DD. The role of citrullinated proteins suggests a novel mechanism in the pathogenesis of multiple sclerosis. Neurochem Res. 2008;32(2):251-256.

47. Pedre X, Mastronardi F, Bruck W, et al. Changed histone acetylation patterns in normal-appearing white matter and early multiple sclerosis lesions. J Neurosci. 2011;31(9):3435-3445

48. Castro K, Casaccia P. Epigenetic modifications in brain and immune cells of multiple sclerosis patients. Mult Scler. 2018;24(1):69-74.

49. Moore LD, Le T, Fan G. DNA methylation and its basic functions. Neuropsychopharmacology. 2013;38(1):23-38.

50. Foley DL, Craig JM, Morley R, et al. Prospects for epigenetic epidemiology. Am J Epidemiol. 2009;169(4):389-400.

51. Urdinguio RG, Sanchez-Mut JV, Esteller M. Epigenetic mechanisms in neuronal diseases: genes, syndromes, and therapies. Lancet Neurol. 2009;8(11):1056-1072.

52. Branco MR, Ficz G, Reik W. Uncovering the role of 5-hydroxymethylcytosine in the epigenome. Nat Rev Genet. 2011;13(1):7-13. 
53. Saitou M, Kagiwada S, Kurimoto K. Epigenetic reprogramming in mouse pre-implantation development and primordial germ cells. Development. 2012;139(1):15-31.

54. Ohtani Fujita N, Fujita T, Aoike A, et al. CpG methylation inactivates the promoter activity of the human retinoblastoma tumor-supressor gene. Oncogene. 1993;8(4):1063-1067.

55. Casaccia-Bonnefil P, Pandozy G, Mastronardi F. Evaluating epigenetic landmarks in the brain of multiple sclerosis patients: A contribution to the current debate on disease pathogenesis. Prog Neurobiol. 2008;86(4):368-378.

56. Illingworth RS, Bird AP. CpG islands-"a rough guide". FEBS Lett. 2009;583(11):1713-1720.

57. Mastronardi FG, Noor A, Wood DD, et al. Peptydil argininedeiminase $2 \mathrm{CpG}$ island in multiple sclerosis white matter is hypometilated. $J$ Neurosci Res. 2007;85(9):2006-2016.

58. Musse AA, Boggs JM, Harauz G. Deimination of membrane-bound myelin basic protein in multiple sclerosis exposes an immunodominant epitope. Proc Natl Acad Sci USA. 2006;103(12):4422-4427.

59. Souza CA, Wood DD, She YM, et al. Autocatalytic cleavage of myelin basic protein: an alternative to molecular mimicry. Biochemistry. 2005;44(38):12905-12913.

60. Ruhrmann S, Ewing E, Piket E, et al. Hypermethylation of MIR21 in $\mathrm{CD}^{+} \mathrm{T}$ cells from patients with relapsing-remitting multiple sclerosis associates with lower miRNA-21 levels and concomitant upregulation of its target genes. Mult Scler. 2017;1:1352458517721356.

61. Li X, Xiao B, Chen XS. DNA Methylation: a New Player in Multiple Sclerosis. Mol Neurobiol. 2017;54(6):4049-4059.

62. Chestnut BA, Chang Q, Price A, et al. Epigenetic regulation of motor neuron cell death through DNA methylation. J Neurosci. 2011;31(46):16619-16636.

63. Siegel SR, Mackenzie J, Chaplin G, et al. Circulating microRNAs involved in multiple sclerosis. Mol Boil Rep. 2012;39(5):6219-6225.

64. Fenoglio C, Ridolfi E, Galimberti D, et al. MicroRNAs as active players in the pathogenesis of multiple sclerosis. Int $\mathrm{J} \mathrm{Mol} \mathrm{Sci}$. 2012;13(10):13227-13239.

65. Bartel DP. MicroRNAs: target recognition and regulatory functions. Cell. 2009;136(2):215-233.

66. Thamilarasan M, Koczan D, Hecker M, et al. MicroRNAs in multiple sclerosis and experimental autoimmune encephalomyelitis. Autoimmun Rev. 2012;11(13):174-179.

67. Liston F, Linterman M, Lu LF. MicroRNA in the adaptive immune system, in sickness and in health. J Clin Immunol. 2010;30(3):339-346.

68. Ha TY. The role of microRNA in regulatory $\mathrm{T}$ cells and immune response. Immune Netw. 2011;11(1):11-41.

69. Allantaz F, Cheng DT, Bergauter T, et al. Expression profiling of human immune cell subsets identifies miRNA - mRNA regulatory relationship correlated with cell type specific expression. PLoS One. 2012;7(1):e29979.

70. Kanwar JR, Mahidhara G, Kanwar RK. MicroRNA in human cancer and chronic inflammatory diseases. Front Biosci (Schol Ed). 2010;2:11131126 .

71. Sontag KC. MicroRNA and deregulated gene expression networks in neurodegeneration. Brain Res. 2010;1338:48-57.
72. Yong VW. Prospects in repair in multiple sclerosis. J Neurol Sci. 2009;277(1):S16-S18.

73. Chang A, Smith MC, Yin R, et al. Neurogenesis in the chronic lesions of multiple sclerosis. Brain. 2008;131(9):2366-2375.

74. Cheng LC, Pastrana E, Tavazoie M, et al. MiR-124 regulates adult neurogenesis in the subventricular zone stem cell niche. Nat Neurosci. 2009;12(4):399-408.

75. Shen Q, Temple S. Fine control: microRNA regulation of adult neurogenesis. Nat Neurosci. 2009;12(4):369-370.

76. Junker A, Hohlfeld R, Meinl E (2011) The emerging role of microRNA in multiple sclerosis. Nat Rev Neurol. 2011;7(1):56-59.

77. Koch MW, Metz LM, Kovalchuk O. Epigenetics and miRNAs in the diagnosis and treatment of multiple sclerosis. Trends Mol Biol. 2013;19(1):23-30.

78. Tufekci KU, Oner MG, Genc S, et al. MicroRNAs and multiple sclerosis. Autoimmun Dis. 2011.

79. Teng G, Hakimpour P, Landgraf P, et al. (2008) MicroRNA-155 is a negative regulator of activation - inducedcytidine deaminidase. Immunity. 2008;28(5):621-629.

80. Du C, Liu C, Kang J, et al. (2009) MicroRNA miR-326 regulates Th17 differentiation and is associated with the pathogenesis of multiple sclerosis. Nat Immunol. 2009;10(12):1252-1259.

81. Otaegu D, Baranzini SE, Armananzas R, et al. Differential micro RNA expression in PBMC from multiple sclerosis patients. PloS One. 2009;4(7):e6309.

82. Lindberg RLP, Hoffmann F, Mehling M, et al. Altered expression of miR-17-5p in $\mathrm{CD}^{+}$lymphocytes of relapsing-remitting multiple sclerosis patients. Eur J Immunol. 2010;40(3):888-898.

83. Cox MB, Cairns MJ, Gandhi KS, et al. (2010) MicroRNAs miR-17 and Mir-20a inhibit T cell activation genes and are under-expressed in MS whole blood. PloS One. 2010;5(8):e12132.

84. Noorbakhsh F, Ellestad KK, Maingat F, et al. Impaired neurosteroid synthesis in multiple sclerosis. Brain. 2011;134(Pt 9):2703-2721.

85. Guerau-de-Arellano M, Smith KM, Godlewski J, et al. MicroRNA dysregulation in multiple sclerosis favours pro-inflammatory T-cellmediated autoimmunity. Brain. 2011;134(Pt 12):3578-3589.

86. Regev K, Paul A, Healy B, et al. Comprehensive evaluation of serum microRNAs as biomarkers in multiple sclerosis. Neurol Neuroimmunol Neuroinflamm. 2016;3(5):e267.

87. Fenoglio C, Cantoni C, de Riz M, et al. Expression and genetic analysis of miRNAs involved in $\mathrm{CD}^{+}$cell activation in patients with multiple sclerosis. Neurosci Lett. 2011;504(1):9-12.

88. Kimura K, Hohjoh H, Fukuoka M, et al. Circulating exosomes suppress the induction of regulatory $\mathrm{T}$ cells via let-7i in multiple sclerosis. Nat Commun. 2018;9(1):17.

89. Naghavian R, Ghaedi K, Kiani-Esfahani A, et al. MiR-141 and miR200a, Revelation of New Possible Players in Modulation of Th17/Treg Differentiation and Pathogenesis of Multiple Sclerosis. PLoS One. 2015;10(5):e0124555.

90. Ahmadian-Elmi M, Bidmeshki Pour A, Naghavian R, et al. MiR-27a and miR-214 exert opposite regulatory roles in Th17 differentiation via mediating different signaling pathways in peripheral blood CD4 ${ }^{+}$ $\mathrm{T}$ lymphocytes of patients with relapsing-remitting multiple sclerosis. Immunogenetics. 2016;68(1):43-54. 